\title{
ON QUANTITATIVE ASPECTS OF THE UNIT SUM NUMBER PROBLEM
}

\author{
CLEMENS FUCHS, ROBERT TICHY, AND VOLKER ZIEGLER
}

\begin{abstract}
We investigate the function $u_{K, S}(m ; q)$, which counts the number of representations of algebraic integers $\alpha$ with $\left|\mathrm{N}_{K / \mathbb{Q}}(\alpha)\right| \leq q$, so that they can be written as sum of exactly $m S$-units of the number field $K$.
\end{abstract}

\section{INTRODUCTION}

The study of the additive structure of units in rings originates in investigations due to Zelinsky [14] back in the fifties of the previous century. Zelinsky showed that every endomorphism of a vector space $V$ over a division ring $D$ can be written as the sum of two automorphisms, unless $D$ is not the field with two elements and $V$ is of dimension 1. Similar results were obtained for other endomorphism rings (for an overview see [13]).

The additive unit structure of maximal orders of number fields have been studied, too. In particular, Jacobson [9] observed that every element in the maximal orders of the number fields $\mathbb{Q}(\sqrt{2})$ and $\mathbb{Q}(\sqrt{5})$ can be written as the sum of distinct units. Later all quadratic, cubic and quartic fields with this property were determined (see $[11,2,3])$. By a combination of results on $S$-unit equations and combinatorial results Jarden and Narkiewicz [10] showed that given a finitely generated domain $B$ of characteristic zero for every positive integer $k$ there exists an $\alpha \in B$ such that $\alpha$ cannot be written as the sum of $k$ units. In view of this observation two problems arise:

The qualitative problem: Which rings of integers are generated by their units?

The quantitative problem: How many non-associated algebraic integers with bounded norm, height, etc. can be written as the sum of $k$ units?

The qualitative problem has been solved for quadratic and complex cubic fields (see $[2,1,12]$. Also some classes of quartic fields have been investigated (see $[8,15])$. Recently, the quantitative problem has been investigated for quadratic fields and some purely quartic fields (see $[8,7]$ ).

This paper is devoted to the quantitative problem. In order to give precise statements we introduce the following counting function:

Definition 1. Let $K$ be a number field, $S$ a finite set of places of $K$ and $U_{K, S}$ the group of $S$-units. As usual, two $S$-integers $\alpha$ and $\beta$ of $K$ are said to be associated if there exists an $S$-unit $\epsilon$ such that $\alpha=\beta \epsilon$ and we write $\alpha \sim \beta$.

1991 Mathematics Subject Classification. 11N45,11D45.

Key words and phrases. sums of units, unit sum number problem.

The third author is supported by the Austrian Science Fund FWF (project numbers: S9611 and S9603). 
We define the counting function $u_{K, S}(n ; q)=u(n ; q)$ as number of equivalence classes $[\alpha]_{\sim}$ such that

$$
N(\alpha):=\prod_{\nu \in S}|\alpha|_{\nu} \leq q, \quad \alpha=\sum_{i=1}^{n} \epsilon_{i}, \quad \epsilon_{i} \in U_{K, S}
$$

and no subsum vanishes.

Note that the function $u(n ; q)$ is well defined, since $N(\alpha)=N(\beta)$ if $\alpha \sim \beta$. Before we state our main theorem we fix the following notation for the rest of the paper. Let $K$ be a number field and $S$ be a finite set of places of $K$ including the archimedian ones and let $|S|=s+1$. By $U_{K, S}$ we denote the group of $S$-units of $K$ and we fix a fundamental system $\epsilon_{1}, \ldots, \epsilon_{s}$ of $S$-units. By $\omega_{K}$ and $\operatorname{Reg}_{K, S}$ we will denote the number of roots of unity and the $S$-regulator of $K$.

Theorem 1. Let $\epsilon>0$. Under the above assumptions we have

$$
u(n ; q)=\frac{c_{n-1, s}}{n !}\left(\frac{\omega_{K}(\log q)^{s}}{\operatorname{Reg}_{K, S}}\right)^{n-1}+o\left((\log q)^{(n-1) s-1+\epsilon}\right)
$$

for all $\epsilon>0$, where the constant $c_{n, s}$ is the volume of

$$
\left\{\left(x_{11}, \ldots, x_{s n}\right) \in \mathbb{R}^{n s}: h\left(x_{11}, \ldots, x_{s n}\right)<1\right\}
$$

with

$$
h\left(x_{11}, \ldots, x_{s n}\right)=\sum_{i=1}^{s} \max \left\{0, x_{i 1}, \ldots, x_{i n}\right\}+\max \left\{0,-\sum_{i=1}^{s} x_{i 1}, \ldots,-\sum_{i=1}^{s} x_{i n}\right\} .
$$

Our theorem is closely related to results due to Everest $[4,5]$ who counted the number of solutions to certain norm inequalities. However, the full result of Everest makes heavy use of many non-elementary results such as theorems on the uniform distribution of sequences, Schmidt's subspace theorem, Baker's theory on linear forms in logarithms, etc. Using the full result would lead us to an error term without $\epsilon$ and a big- $O$ instead of a small-o.

In order to give the reader more insight we sketch Everst's proof and try to avoid most of the non elementary steps. Unfortunately, we cannot avoid an application of Schmidt's subspace theorem (see Lemma 3, second statement). However, the interested reader is advised to study Everest's papers for a full account to the techniques described below.

In the next section we introduce some notations that will help us in section 3 to state Everest's results and to sketch their proofs. Applying these results we will prove our main theorem in section 4 . Since the definition of the constant $c_{n, s}$ is not very illuminating we discuss an estimate for $c_{n, s}$ in the last section.

\section{Some Notations}

We want to study $S$-unit representations with small norm and so we introduce the following function. Let $\underline{x}=\left(1, x_{2}, \ldots, x_{n}\right) \in U_{K, S}^{n}$ then we consider the function

$$
N_{K, S}(\underline{x})=N(\underline{x})=\prod_{\nu \in S}\left|1+x_{2}+\cdots+x_{n}\right|_{\nu}
$$

and its related counting function

$$
N_{K, S}(q)=N(q)=\sharp\left\{\underline{x} \in U_{K, S}^{n}: N(\underline{x})<q\right\} .
$$


where no subsum of $1+x_{2}+\cdots+x_{n}$ vanishes. We are also interested in the following variant: Let $\underline{c}=\left(c_{1}, \ldots, c_{n}\right) \in\left(K^{*}\right)^{n}$ be fixed, we define

$$
N_{\underline{c}}(\underline{x})=\prod_{\nu \in S}\left|c_{1}+c_{2} x_{2}+\cdots+c_{n} x_{n}\right|_{\nu}
$$

and the corresponding counting function

$$
N_{\underline{c}}(q)=\sharp\left\{\underline{x} \in U_{K, S}^{n}: N_{\underline{c}}(\underline{x})<q\right\},
$$

where no subsum of $c_{1}+c_{2} x_{2}+\cdots+c_{n} x_{n}$ vanishes.

As we will see in the next section these functions are closely related to the height functions

$$
H_{\nu}(\underline{x})=\max \left\{0,\left|x_{i}\right|_{\nu} ; i=1, \ldots, n\right\}
$$

and

$$
H(\underline{x})=\prod_{\nu \in S} \max \left\{0,\left|x_{i}\right|_{\nu} ; i=1, \ldots, n\right\}=\prod_{\nu \in S} H_{\nu}(\underline{x})
$$

and its counting function

$$
H(q)=\sharp\left\{\underline{x} \in U_{K, S}^{n}: H(\underline{x})<q\right\} .
$$

As will be shown later the following set $U_{0} \subset U_{K, S}^{n}$ will yield the main contribution to $H(q)$. We write $H_{\nu}^{*}$ to be the second largest member of the set $\left\{0,\left|x_{i}\right|_{\nu}\right\}$. Given reals $A_{\nu}>0, B_{\nu}>1$, define

$$
U_{0}=\left\{\underline{x} \in U_{K, S}^{n}: \forall \nu \in S, \frac{H_{\nu}^{*}(\underline{x})}{H_{\nu}(\underline{x})}<A_{\nu} e^{-B_{\nu} \log \log H(\underline{x})}\right\} .
$$

Next we define the counting function

$$
H_{0}(q)=\sharp\left\{\underline{x} \in U_{0}: H(\underline{x})<q\right\} .
$$

Assume for the rest of the paper that $A_{\nu}$ and $B_{\nu}$ are fixed numbers. This choice does not effect the asymptotics but rather the constants in the error terms.

\section{Sums of $S$-units}

In this section we adapt the results and techniques due to Everest $[4,5]$ to our case. We start with

\section{Lemma 1.}

$$
H(q)=c_{n, s}\left(\frac{\omega_{K}(\log q)^{s}}{\operatorname{Reg}_{K, S}}\right)^{n-1}+O\left((\log q)^{(n-1) s-1}\right) .
$$

Proof. Let $x_{i}$ be $S$-units and write $x_{i}=\zeta_{i} \epsilon_{1}^{n_{i 1}} \cdots \epsilon_{s}^{n_{i s}}$ for $i=1, \ldots, n$. Taking logarithms of the absolute values $|\cdot|_{\nu}$ with $\nu \in S$ and recalling the definition of $H(\underline{x})$ we see that the function $H(q)$ essentially counts the number of points $\underline{n}=\left(n_{11}, \ldots, n_{n s}\right) \in \mathbb{Z}^{n s}$ that fulfill the inequality

$$
\sum_{i=1}^{s+1} \max \left\{0, L_{i}^{(j)}(\underline{n})\right\}<\log q,
$$

where

$$
L_{i}^{(j)}(\underline{n})=n_{i 1} \log \left|\epsilon_{i}\right|_{\nu_{j}}+\cdots+n_{i s} \log \left|\epsilon_{s}\right|_{\nu_{j}}=\log \left|x_{i}\right|_{\nu_{j}},
$$


where $S=\left\{\nu_{1}, \ldots, \nu_{s+1}\right\}$. A linear transformation of determinant $\operatorname{Reg}_{K, S}^{-1}$ turns the $L_{i}^{(j)}(\underline{n})$ into

$$
\tilde{L}_{i}^{(j)}(\underline{n})=\left\{\begin{array}{lr}
n_{i j} & \text { for } j \leq s \\
-\sum_{k=1}^{s} n_{i k} & \text { for } j=s+1 .
\end{array}\right.
$$

Note that the number of lattice points in a polyhedron of dimension $n$ is its volume plus an error term of the size about the $n-1$ dimensional volume of its faces. Bearing in mind the linear transformation and the fact that the number of lattice points are points counted by $H(q)$ up to multiplication by roots of unity we obtain the lemma.

For the function $H_{0}(q)$ we obtain (see [5, Proposition 1]).

Lemma 2.

for all $\epsilon>0$.

$$
H_{0}(q)=H(q)+o\left((\log q)^{(n-1) s-1+\epsilon}\right)
$$

Note that the proof of this lemma does not need any deep results and can be achieved by considering Dirichlet series (see also [4, Lemma 3]).

Next, we want to establish a relation between $H(x)$ and $N(x)$ (see [4, Lemma 6]).

Lemma 3. (1) For all $\underline{x} \in U_{0}$ we have $\log N(x)=\log H(x)+O(1 / \log (H(x)))$.

(2) For all $\underline{x} \in U_{K, S}$ such that no subsum of $1+x_{2}+\ldots+x_{n}$ vanishes, there exists a constant $\theta>0$ such that $\log N(x)>\theta \log H(x)$

Proof. We have

$$
\begin{aligned}
\log N(\underline{x}) & =\sum_{\nu \in S} \log \left|1+x_{2}+\cdots+x_{n}\right|_{\nu} \\
& =\sum_{\nu \in S} \log H_{\nu}(\underline{x})+\sum_{\nu \in S} \log \left(1+O\left(\frac{H_{\nu}^{*}(\underline{x})}{H_{\nu}(\underline{x})}\right)\right)
\end{aligned}
$$

because of the definition of $U_{0}$ the first statement is proved. The second statement is a consequence of Schmidt's subspace theorem (see [6, Theorem 2] and in the case of $K$ a quadratic field see also [7, Lemma 3])

Now we establish the main result of this section (see also [5, Theorem 1]):

\section{Proposition 1.}

$$
N(q)=c_{n, s}\left(\frac{\omega_{K}(\log q)^{s}}{\operatorname{Reg}_{K, S}}\right)^{n-1}+o\left((\log q)^{(n-1) s-1+\epsilon}\right)
$$

for all $\epsilon>0$.

Proof. We break up to counting function into two parts:

$$
\begin{aligned}
N(q) & =\sharp\left\{\underline{x}: N(\underline{x})<q, x \in U_{0}\right\}+\sharp\left\{\underline{x}: N(\underline{x})<q, x \notin U_{0}\right\} \\
& =N_{1}(q)+N_{2}(q)
\end{aligned}
$$

For the second part we obtain by Lemma 3

$$
N_{2}(q)=\sharp\left\{\underline{x}: H(\underline{x})^{\theta}<q, x \notin U_{0}\right\}=o\left((\log q)^{(n-1) s-1+\epsilon}\right),
$$


where the last equality is obtained by a combination of the Lemmas 1 and 2 . Since those $\underline{x}$ that satisfy $H(\underline{x})<(\log q)^{1 / 2}$ are only $O\left((\log q)^{(n-1) s / 2}\right)$ in number, we may assume $H(\underline{x})>(\log q)^{1 / 2}$. So we obtain

$$
\begin{aligned}
N_{1}(q) & =\sharp\left\{\underline{x} \in U_{0}: \log (H(\underline{x}))<\log q+O(1 / \sqrt{\log q})\right\} \\
& =H_{0}\left(q^{1+O(1 / \sqrt{\log q})}\right) \\
& =H\left(q^{1+O(1 / \sqrt{\log q})}\right)+o\left(((1+O(1 / \sqrt{\log q})) \log q)^{s(n-1)-1+\epsilon}\right) \\
& =c_{n, s}\left(\frac{\omega_{K}(\log q)^{s}}{\operatorname{Reg}_{K, S}}\right)^{n-1}+o\left((\log q)^{(n-1) s-1+\epsilon}\right)+O\left(\log q^{(n-1) s-3 / 2}\right) \\
& =c_{n, s}\left(\frac{\omega_{K}(\log q)^{s}}{\operatorname{Reg}_{K, S}}\right)^{n-1}+o\left((\log q)^{(n-1) s-1+\epsilon}\right)
\end{aligned}
$$

Corollary 1. Assume for all $\nu \in S$ that $\log \left|\epsilon_{1}\right|_{\nu}, \ldots, \log \left|\epsilon_{s}\right|_{\nu}$ generate a $\mathbb{Q}$-space of dimension at least 2 . Then

$$
N(q)=c_{n, s}\left(\frac{\omega_{K}(\log q)^{s}}{\operatorname{Reg}_{K, S}}\right)^{n-1}+O\left((\log q)^{(n-1) s-1}\right)
$$

Proof. Combine Everest's result [5, Theorem 1] with Proposition 1.

At the end of the section we want to state Everest's result [5, Theorem 1] for $N_{c}(q)$ which stated in this form may be obtained analogously to the proof of Proposition 1, i.e. the only non elementary part is the proof of the second statement of Lemma 3.

Proposition 2. For all $\underline{c} \in\left(K^{*}\right)^{n}$ we have

$$
N_{\underline{c}}(q)=O\left((\log q)^{(n-1) s}\right) .
$$

\section{The number of $S$-Unit Representations}

This section is devoted to the proof of our main Theorem 1. Assume $\alpha=$ $x_{1}+\ldots+x_{n}$ can be written as a sum of $S$-units, where

$$
x_{i}=\zeta_{i} \epsilon_{1}^{n_{i 1}} \cdots \epsilon_{s}^{n_{i s}}
$$

for $i=1, \ldots, n$. Then we can write $\alpha=1+x_{2}+\cdots+x_{n}$, with $0=n_{1 s} \leq n_{2 s} \leq$ $\cdots \leq n_{n s}$, since we are interested only in equivalence classes of associated integers. Let us denote by $u_{0}(n ; q)$ the number of representations, where all units are pairwise distinct. Then we have $u_{0}(n ; q)=N(q) / n !-r$, where $r$ is the number of integers $\alpha$ that have two distinct representations as sums of $n$ distinct $S$-units. As shown in [7, Lemma 2] $r$ depends only on $n$ and $S$ but not on $q$. The factor $1 / n$ ! comes from the fact that the order of the $x_{i}$ does not matter in the computation of $u_{0}(n ; q)$.

On the other hand we have

$$
u(n ; q)=u_{0}(n ; q)+\sum_{\underline{c}} \frac{N_{\underline{c}}(q)}{l(\underline{c}) !}+O(1),
$$

where the $O(1)$ comes from the $\alpha^{\prime} s$ with two distinct representations and the sum runs over all $\underline{c}=\left(c_{1}, \ldots, c_{m}\right) \in \mathbb{Z}^{m}$ with $c_{i} \geq 1$ and $c_{1}+\cdots+c_{m}=n$ and 
$\left(c_{1}, \ldots, c_{m}\right) \neq(1, \ldots, 1)$, i.e. $m<n$ for all $\underline{c}$ 's appearing in the summation. Moreover we write $l(\underline{c})=m$. Applying the Propositions 1 and 2 to formula (2) proves our main Theorem 1.

\section{SOME RESUlts FOR $c_{n, s}$}

In this section we want to discuss the quantity $c_{n, s}$. For small values of $s$ and $n$ it is possible to compute the quantity $c_{n, s}$ directly. We did this for all pairs $(n, s)$ with $n+s \leq 6$ (see Table 1$)$.

TABLE 1. Values for $c_{n, s}$

\begin{tabular}{|c||c|c|c|c|c|}
\hline$s \backslash n$ & 1 & 2 & 3 & 4 & 5 \\
\hline \hline 1 & 2 & 3 & 4 & 5 & 6 \\
\hline 2 & 3 & $\frac{15}{4}$ & $\frac{7}{2}$ & $\frac{45}{16}$ & \\
\hline 3 & $\frac{10}{3}$ & $\frac{7}{3}$ & $\frac{55}{54}$ & & \\
\hline 4 & $\frac{35}{12}$ & $\frac{275}{32}$ & & & \\
\hline 5 & $\frac{21}{10}$ & & & & \\
\hline
\end{tabular}

Furthermore, we can prove the following:

Lemma 4. (1) $c_{n, 1}=n+1$;

(2) $c_{1, s}=\frac{4^{s} \Gamma(s+1 / 2)}{\sqrt{\pi} \Gamma(s+1)^{2}}$

(3) $\frac{2^{n s}}{(n s) !}<c_{n, s}<2^{n s}$;

Proof. First we consider the case $s=1$, i.e. we have to compute the volume of

$$
\mathcal{B}_{n, 1}:=\left\{\left(x_{1}, \ldots, x_{n}\right) \in \mathbb{R}^{n}: \max \left\{0, x_{1}, \ldots, x_{n}\right\}+\max \left\{0,-x_{1}, \ldots,-x_{n}\right\}\right\} .
$$

Assume the following holds:

$$
x_{1}>x_{2}>\cdots>x_{r}>0>x_{r+1}>\cdots>x_{n}
$$

with $0<r<n$. Then we have $x_{1}-x_{n}<1$. Note that the case $r=0$ respectively $r=n$ yields the volume of a simplex with rectangular edges of length 1 hence volume $1 / n$ !. In the other cases the volume is

$$
\begin{aligned}
& \int_{x_{1}=0}^{1} \int_{x_{n}=x_{1}-1}^{0} \int_{x_{2}=0}^{x_{1}} \cdots \int_{x_{r}=0}^{x_{r-1}} \int_{x_{n-1}=x_{n}}^{0} \cdots \int_{x_{r+1}=x_{r+2}}^{0} 1 d x_{r+1} \cdots d x_{n-1} d x_{r} \cdots d x_{2} d x_{n} d x_{1}= \\
& \int_{x_{1}=0}^{1} \int_{x_{n}=x_{1}-1}^{x_{x_{2}-1}} \int_{x_{2}=0}^{x_{1}} \cdots \int_{x_{r}=0}^{x_{r}} \frac{\left(-x_{n}\right)^{n-r-1}}{(n-r-1) !} d x_{r} \cdots d x_{2} d x_{n} d x_{1}= \\
& \int_{x_{1}=0}^{1} \int_{x_{n}=x_{1}-1}^{0} \frac{\left(-x_{n}\right)^{n-r-1} x_{1}^{r-1}}{(n-r-1) !(r-1) !} d x_{n} d x_{1}= \\
& \int_{x_{1}=0}^{1} \frac{\left(1-x_{1}\right)^{n-r} x_{1}^{r-1}}{(n-r) !(r-1) !} d x_{1}=\frac{1}{n !}
\end{aligned}
$$

So any case (also the cases $r=0$ and $r=n$ ) yields the volume $1 / n$ !. Since there are $(n+1)$ ! cases we obtain $c_{n, 1}=(n+1) ! / n !=n+1$. 
Now we consider the case $n=1$. In this case we have to compute the volume of

$$
\mathcal{B}_{1, s}:=\left\{\left(x_{1}, \ldots, x_{s}\right) \in \mathbb{R}^{s}: h\left(x_{1}, \ldots, x_{s}\right)<1\right\}
$$

where $h\left(x_{1}, \ldots, x_{s}\right):=\max \left\{0, x_{1}\right\}+\cdots+\max \left\{0, x_{s}\right\}+\max \left\{0,-x_{1}-\cdots-x_{s}\right\}$. Let us assume $x_{1}, \ldots, x_{r}>0$ and $x_{r+1}, \ldots, x_{s} \leq 0$. Then $h\left(x_{1}, \ldots, x_{s}\right)=x_{1}+\cdots+x_{r}$ if $-x_{1}-\ldots-x_{s} \leq 0$ and $h\left(x_{1}, \ldots, x_{s}\right)=-x_{r+1}-\cdots-x_{s}$ otherwise. In the second case we may make the coordinate change $y_{i}=-x_{i}$ with $1 \leq i \leq s$ and obtain the first case with $s-r$ instead of $r$. Therefore we consider only the case $x_{1}, \ldots, x_{r}>0$, $x_{r+1}, \ldots, x_{s} \leq 0$ and $x_{1}+\ldots+x_{s}>0$. Then we have to compute the following integral:

$$
\begin{aligned}
& \int_{x_{1}=0}^{1} \ldots \int_{x_{r}=0}^{1-\sum_{i=1}^{r-1} x_{i}} \int_{x_{r+1}=-\sum_{i=1}^{r} x_{i}}^{0} \cdots \int_{x_{s}=-\sum_{i=1}^{s-1} x_{i}}^{0} 1 d x_{s} \cdots d x_{1}= \\
& \int_{x_{1}=0}^{1} \ldots \int_{x_{r}=0}^{1-\sum_{i=1}^{r-1} x_{i}} \frac{\left(\sum_{i=1}^{r} x_{i}\right)^{s-r}}{(s-r) !} d x_{r} \cdots d x_{1}= \\
& \int_{x_{1}=0}^{1} \ldots \int_{x_{r-1}=0}^{1-\sum_{i=1}^{r-2} x_{i}} \frac{1}{(s-r+1) !}-\frac{\left(\sum_{i=1}^{r-1} x_{i}\right)^{s-r+1}}{(s-r+1) !} d x_{r-1} \cdots d x_{1}= \\
& \frac{1}{(s-r+1) !(r-1) !}-\int_{x_{1}=0}^{1} \ldots \int_{x_{r-1}=0}^{1-\sum_{i=1}^{r-2} x_{i}} \frac{\left(\sum_{i=1}^{r-1} x_{i}\right)^{s-r+1}}{(s-r+1) !} d x_{r-1} \cdots d x_{1}= \\
& \sum_{j=1}^{r} \frac{(-1)^{j+1}}{(s-r+j) !(r-j) !}=\frac{1}{(s-r) !(r-1) ! s}
\end{aligned}
$$

All together we obtain

$$
c_{n, s}=\sum_{j=0}^{s} \frac{2\left(\begin{array}{c}
s \\
j
\end{array}\right)}{(s-r) !(r-1) ! s}=\frac{4^{s} \Gamma(s+1 / 2)}{\sqrt{\pi} \Gamma(s+1)^{2}} .
$$

We are left to prove the last statement of the lemma. Since we have $\left|x_{i, j}\right|<1$ for $1 \leq i \leq n$ and $1 \leq j \leq s$ the upper bound is established. For the lower bound we use the fact that all $x_{i j}$ satisfying

$$
\sum_{i=1}^{n} \sum_{j=1}^{s}\left|x_{i, j}\right|<1
$$

lie in the body that defines $c_{n, s}$.

Remark 1. With a little more effort it would be possible to show sharper bounds for $c_{n, s}$. We abandon to do so because the constant does not effect the asymptotics of $u(n ; q)$ which is our main concern in this paper. 
Let us consider the result for $s=1$, i.e. let us consider the unit group of a real quadratic field, complex cubic field or totally complex quartic field $K$. This yields

$$
u(n ; q)=\frac{1}{(n-1) !}\left(\frac{\omega_{K}(\log q)}{\log |\eta|}\right)^{n-1}+o\left((\log q)^{(n-2+\epsilon}\right)
$$

where $\eta$ is a fundamental unit of $K$ with $|\eta|>1$. Moreover, note that the number of roots of unity $\omega_{K}=2$ if $K$ is quadratic or a cubic field or a quartic field containing neither $i$ nor $\frac{1+i \sqrt{3}}{2}$. In particular, for $K$ a real quadratic field we get

$$
u(n ; q)=\frac{1}{(n-1) !}\left(\frac{2(\log q)}{\log \eta}\right)^{n-1}+o\left((\log q)^{(n-2+\epsilon}\right)
$$

which is an improvement of the result obtained in [7].

\section{REFERENCES}

[1] N. Ashrafi and P. Vámos. On the unit sum number of some rings. The Quarterly Journal of Mathematics, 56(1):1-12, 2005.

[2] P. Belcher. Integers expressible as sums of distinct units. Bull. Lond. Math. Soc., 6:66-68, 1974.

[3] P. Belcher. A test for integers being sums of distinct units applied to cubic fields. J. Lond. Math. Soc., II. Ser., 12:141-148, 1976.

[4] G. R. Everest. A "Hardy-Littlewood" approach to the S-unit equation. Compositio Math., 70(2):101-118, 1989.

[5] G. R. Everest. Counting the values taken by sums of $S$-units. J. Number Theory, 35(3):269286, 1990.

[6] J.-H. Evertse. On sums of S-units and linear recurrences. Compositio Math., 53(2):225-244, 1984.

[7] A. Filipin, R. Tichy, and V. Ziegler. On the quantitative unit sum number problem - An application of the subspace theorem. to appear in Acta Arithmetica.

[8] A. Filipin, R. Tichy, and V. Ziegler. The additive unit structure of purely quartic complex fields. to appear in Functiones et Approximationes.

[9] B. Jacobson. Sums of distinct divisors and sums of distinct units. Proc. Am. Math. Soc., 15:179-183, 1964.

[10] M. Jarden and W. Narkiewicz. On sums of units. Monatsh. Math., 150(4):327-336, 2007.

[11] J. Śliwa. Sums of distinct units. Bull. Acad. Pol. Sci., 22:11-13, 1974.

[12] R. Tichy and V. Ziegler. Units generating the ring of integers of complex cubic fields. Colloquim mathematicum, 109(1):71-83, 2007.

[13] P. Vámos. 2-good rings. The Quarterly Journal of Mathematics, 56(3):417-430, 2005.

[14] D. Zelinsky. Every linear transformation is a sum of nonsingular ones. Proc. Am. Math. Soc., 5:627-630, 1954.

[15] V. Ziegler. The unit sum number of complex biquadratic fields. to appear in Glasnik Matematički. 
C. FuChS

ETH ZURICH

Department of Mathematics

RAEMISTRASSE 101

8092 ZURICH, SWITZERLAND

E-mail address: clemens.fuchs@math.ethz.ch

R. TICHY

Graz University of Technology

INSTITUTE FOR ANALYSIS AND COMPUTATIONAL NUMBER THEORY, STEYRERGASSE 30 ,

A-8010 Graz, Austria

E-mail address: tichy@tugraz.at

V. ZIEGLER

Graz University of TeChNology

INSTITUTE FOR ANALYSIS AND COMPUTATIONAL NUMBER THEORY,

STEYRERGASSE 30,

A-8010 Graz, Austria

E-mail address: ziegler@finanz.math.tugraz.at 